\title{
RATE-DEPENDENT PULL-OFF FORCE - AN EXPERIMENTAL APPROACH TO IDENTIFY THE SEPARATION MODE IN MICROCONTACTS
}

\author{
L. Chen ${ }^{1}$, Y. Du ${ }^{2}$, N.E.McGruer ${ }^{3}$, and G.G.Adams ${ }^{3}$ \\ ${ }^{1}$ RF Micro Devices, Inc., Charlotte, North Carolina, USA \\ ${ }^{2}$ Qualcomm, San Jose, California, USA \\ ${ }^{3}$ Northeastern University, Boston, Massachusetts, USA
}

\begin{abstract}
In this paper, we report our observation of rate-dependence of the pull-off force in microcontacts. Two rate-dependent features have been observed during contact cycling tests. One is a larger pull-off force at a low rate (LFLR), and the other is a larger pulloff force at a high rate (LFHR). Further investigation using SEM demonstrates that these two rate-dependent features can be related to two distinct separation modes, i.e. brittle and ductile modes. LFLR behavior may be due to surface effects in brittle separation, whereas LFHR behavior could be due to viscous effects in ductile separation. By monitoring the rate-dependency of the pull-off force, the separation mode could be identified.
\end{abstract}

\section{INTRODUCTION}

Applications of MEMS switches generally require reliable closing and opening of microcontacts over many billions of contact cycles [1]. During operation, topographical and/or material changes can alter the contact resistance and contact adhesion, leading to fail-to-close or fail-to-open switch failures. In order to study microcontact evolution, a scanning probe microscope (SPM) based contact tester was built [2]. Contact tests were performed between hemispherical contact bumps and planar substrates. The contact bumps were microfabricated on silicon test cantilevers. Both the bumps and substrates were coated with the contact test material. Using this contact tester, we have studied different contact materials [3], contact adhesion [4], and separation modes [5]. However in the previous studies, we were unable to identify the separation mode during the cycling test.

In general, there are two separation modes in microcontacts. One is the brittle mode in which rupture occurs abruptly with little or no plastic deformation. The other is the ductile mode in which rupture occurs more gradually with significant plastic deformation. Figure 1 shows typical differences in the force displacement curves for the two separation modes.

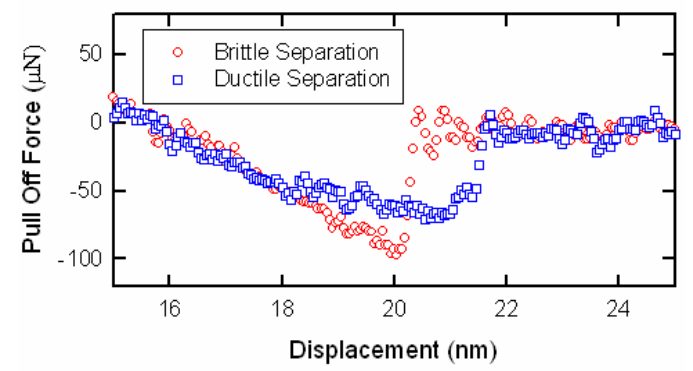

Figure 1: Force displacement curves for the two separation modes. Ductile separation shows a change in slope of the force vs. displacement characteristic due to plastic deformation and a gradual separation. Brittle separation shows a constant slope followed by a sudden separation with ringing of the cantilever. Both force-displacement curves were measured at an unloading rate of $35 \mu \mathrm{m} / \mathrm{s}$.
Depending on the separation mode, the surface morphology of the microcontacts can change, leading eventually to large changes in the pull-off force (the force needed to separate the contacts) [4, 5]. In this paper we report our observations of the rate-dependence of the pull-off force. In particular we find that the magnitude of the pull-off force depends on the cycling and separation rates, and this rate-dependence is related to the separation mode. This behavior allows the separation mode to be identified during testing without SEM observation.

\section{EXPERIMENT}

To study the rate-dependent pull-off force, a $250 \mathrm{~nm}$ gold thin film was sputtered on the stiff test cantilever and also on the mating planar substrate. The tests were performed in laboratory air with a relative humidity of $30 \%$ to $40 \%$. The cycling test uses an additional piezo-actuator to achieve a high cycling rate. The pulloff force is measured using the SPM. The rate-dependent pull-off force is studied by: (i) comparing the pull-off force at two cycling rates $(0.5 \mathrm{~Hz}$ and $300 \mathrm{~Hz})$; (ii) comparing rate-dependence at different loading; (iii) comparing the pull-off force by sweeping the cycling rate between $0.5 \mathrm{~Hz}$ and $1000 \mathrm{~Hz}$; and, (iv) comparing pull-off force at different unloading rates.
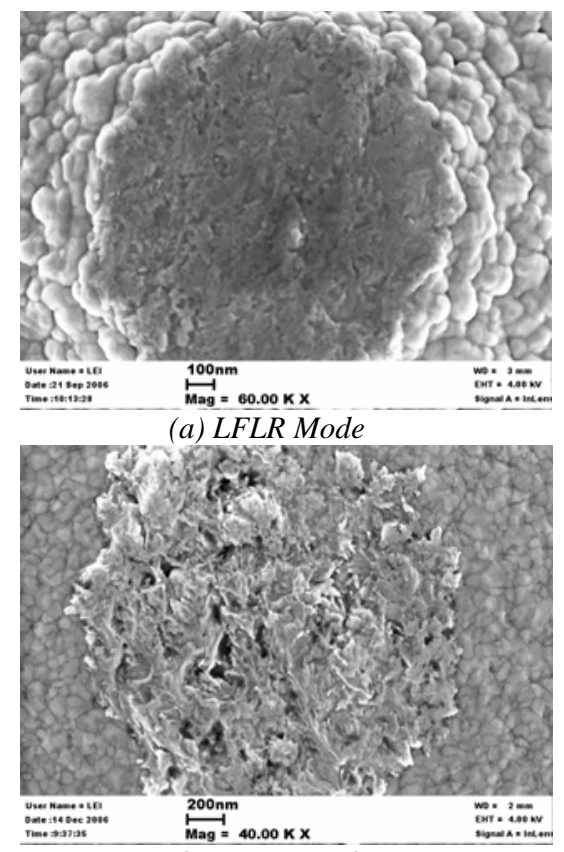

(b) LFHR Mode

Figure 2: SEM micrograph of gold contact bumps with a maximum loading force of $200 \mu N$. (a) For the bump with a radius of curvature of $4 \mu \mathrm{m}$, the pull-off force at $0.5 \mathrm{~Hz}$ is $172 \pm 11 \mu \mathrm{N}$ and the pull-off force at $300 \mathrm{~Hz}$ is $94 \pm 9.5 \mu \mathrm{N}$; (b) For the bump with a radius of curvature of $50 \mu \mathrm{m}$, the pull-off force at $0.5 \mathrm{~Hz}$ is $130 \pm 9.7 \mu \mathrm{N}$, and the pull-off force at $300 \mathrm{~Hz}$ is $165 \pm 7.2 \mu \mathrm{N}$. 


\section{Rate Switching Test}

We first compared the pull-off force at two cycling rates $(0.5 \mathrm{~Hz}$ and $300 \mathrm{~Hz})$, by suddenly switching from one frequency to the other. The maximum contact force was controlled at $200 \mu \mathrm{N}$ during the cycling. After the test, the samples were inspected in the SEM. For gold contacts, we observed two distinctive ratedependent features in the pull-off force. One feature shows a larger pull-off force at low cycling rates (LFLR). The other feature shows a larger pull-off force at high cycling rates (LFHR). SEM observation shows plastically flattened features (Figure 2a), at the surface of the contact in the LFLR mode. However, in the LFHR mode, ductile necks and/or signs of material transfer are always seen (Figure 2b).

\section{Force Sweeping Test}

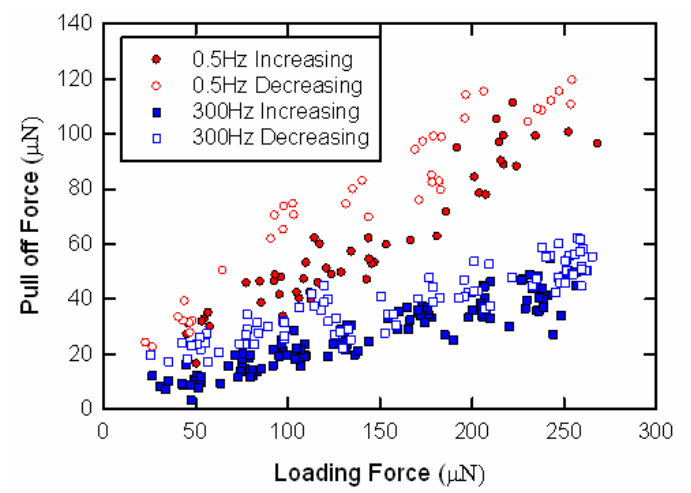

(a)

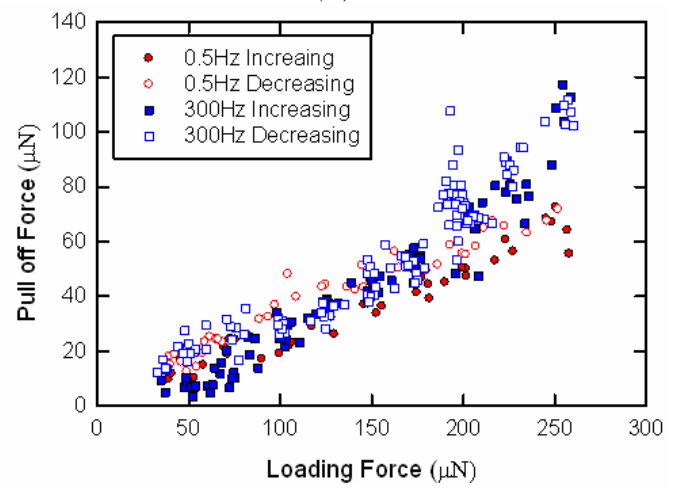

(b)

Figure 3: Rate-dependent pull-off force for separation with (a) larger force at low rate (LFLR), and (b) larger force at high rate (LFHR).

We then investigated the loading effects on the rate related pull-off force. The loading force was manually changed while cycling the contact. For one load sweep, the loading was increased from 0 to $250 \mu \mathrm{N}$, and then decreased back to zero. Each sweeping process took about 120 180 seconds (without strict control). Two cycling rates were used, $0.5 \mathrm{~Hz}$ and $300 \mathrm{~Hz}$. The load sweep was first performed at $0.5 \mathrm{~Hz}$, and then followed by the $300 \mathrm{~Hz}$ test. The samples are gold bumps with radius of curvature of $15 \mu \mathrm{m}$. The pull-off force was recorded during the cycling. The test results are shown in Figure 3. In both LFLR mode and LFHR mode, the pulloff force increases with an increase in the loading force. However, the response of the pull-off force is different in each mode according to the change in the loading.

In LFLR mode, as shown in Figure 3 (a), a larger pull-off force is measured at $0.5 \mathrm{~Hz}$ than at $300 \mathrm{~Hz}$. The difference between the two rates increases from $20 \mu \mathrm{N}$ at a loading of $150 \mu \mathrm{N}$ to $40 \mu \mathrm{N}$ at a loading of $250 \mu \mathrm{N}$. It is also noted that the pull-off force, as the loading is decreased, is slightly higher than the force as the loading is increased. This is probably due to the wear of the surface film during the test.

On the other hand, in LFHR mode, as shown in Figure 3 (b), the pull-off force measured at the two rates $(0.5 \mathrm{~Hz}$ and $300 \mathrm{~Hz})$ are almost the same for a loading force less than $200 \mu N$. However, at loading forces greater than $200 \mu \mathrm{N}$, the pull-off force measured at $300 \mathrm{~Hz}$ becomes larger than the force measured at $0.5 \mathrm{~Hz}$. For a loading force of $250 \mu \mathrm{N}$, there is a $40 \mu \mathrm{N}$ difference in the pull-off force between the two cycling rates. It is also noted that the increase of the pull-off force is more random in the LFHR mode than in the LFLR mode.

\section{Rate Sweeping Test}

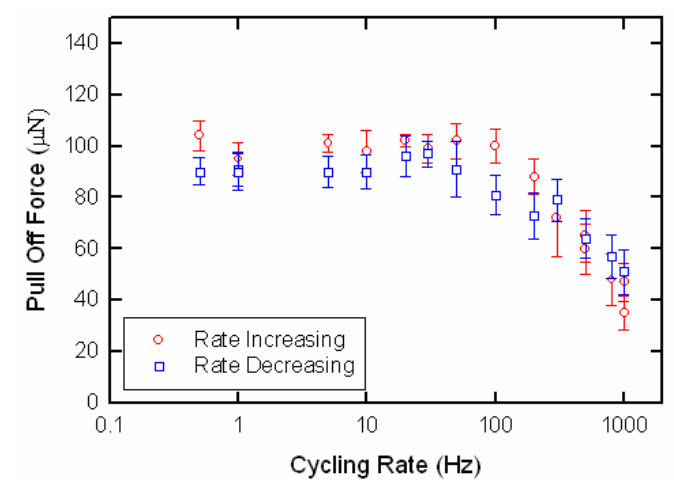

(a)

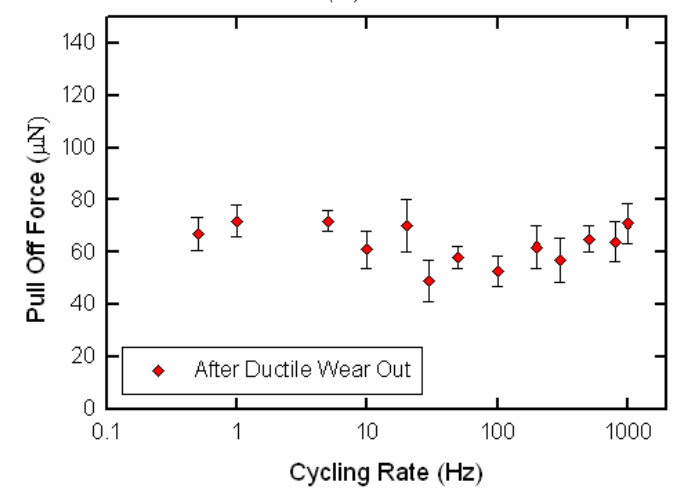

(b)

Figure 4: The rate-dependent response of the pull-off force by sweeping the cycling rate. The maximum loading force is maintained at $200 \mu N$. (a) is the pull-off force response for less than $10^{5}$ cycles (LFLR); (b) is the response after $10^{6}$ cycles of testing.

We also studied the rate-dependent pull-off force properties by sweeping the cycling rate from $0.5 \mathrm{~Hz}$ to $1000 \mathrm{~Hz}$. The sample used for test was a gold bump with a radius of curvature of $15 \mu \mathrm{m}$. During the test, the maximum loading force was controlled at $200 \mu \mathrm{N}$. The cycling rate was increased from $0.5 \mathrm{~Hz}$ to $1000 \mathrm{~Hz}$, and then decreased back to $0.5 \mathrm{~Hz}$. During the rate sweep, 13 different cycling rates were tested. At each cycling rate, the sample was cycled for 30 seconds for pull-off force measurement. The sampling rate for data acquisition was $500 \mathrm{k} / \mathrm{s}$ for cycling rates of $100 \mathrm{~Hz} \sim 1000 \mathrm{~Hz} 50 \mathrm{k} / \mathrm{s}$ for the rates of $10 \sim 100 \mathrm{~Hz}$, and $2 \mathrm{k} / \mathrm{s}$ for the rates of $0.5 \mathrm{~Hz} \sim 10 \mathrm{~Hz}$ respectively.

One such result is shown in Figure 4. A LFLR mode is clearly 
demonstrated in Figure 4 (a). It is shown that the pull-off force is constant at a low cycling rate, and starts to decrease for cycling rates larger than $100 \mathrm{~Hz}$. The pull-off force changed from $100 \mu \mathrm{N}$ measured at $0.5 \mathrm{~Hz}$ to $40 \mu \mathrm{N}$ measured at $1000 \mathrm{~Hz}$. A rate of $100 \mathrm{~Hz}$ appears to be a transition point during the rate sweeping test. It is also noted that such a transition feature can disappear after a certain number of cycles. Figure 4(b) shows the same rate sweeping test result from the same contact after being cycled for $10^{6}$ times. There is no clear rate-dependent feature, and the pull-off forces measured at different rates were similar. After this test, the contact surface was inspected in the SEM. The SEM images show damage or material transfer on the contact surface. Surface wear could be the reason for the disappearance of the LFLR features.

We found that it was not easy to observe the LFHR mode during the rate sweeping test. However, we were able to characterize the LFHR mode by using the unloading rate test.

\section{Unloading Rate Test}

The unloading rate test is performed by using a series of pulse signals. Each signal has the same rise time $(10 \mathrm{~ms})$, the same resting time $(10 \mathrm{~ms})$, but different unloading times. The unloading rate is related to the unloading time. A longer unloading time provides a lower unloading rate. By varying unloading time, eight unloading rates, from $0.05 \mu \mathrm{m} / \mathrm{s}$ to $50 \mu \mathrm{m} / \mathrm{s}$, have been tested. At each unloading rate, the pull-off force is measured with two different maximum loading forces $(100 \mu \mathrm{N}$ and $200 \mu N)$. Some results are shown in Figure 5. It is clear that a LFHR feature was recorded. At a maximum loading of $200 \mu \mathrm{N}$, the pull-off force increased with an increase of the unloading rate. However, such a trend is not clear at a lower loading $(100 \mu \mathrm{N})$.

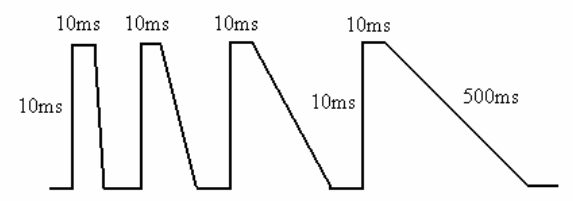

(a)

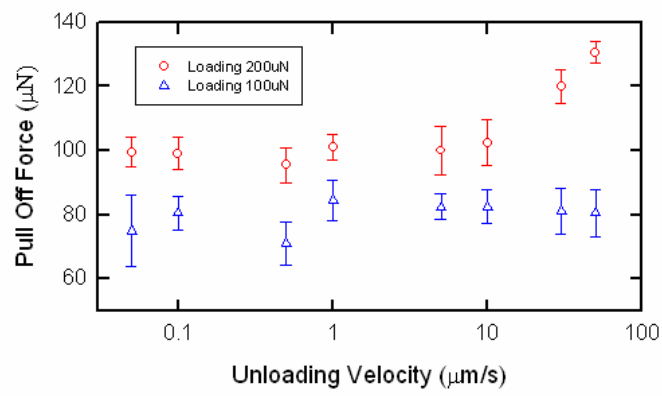

(b)

Figure 5: Pull-off force test with different unloading velocity. (a) The driving waveform for the test. Each pulse with the same loading time and the same time in contact, but with different unloading time; (b) measured unloading rate-dependent pull-off force in the LFHR mode.

\section{DISCUSSION}

According to our rate-dependent pull-off force contact tests, there are two different rate-dependent features that have been identified. One feature shows a larger pull-off force at low cycling rates (LFLR). The other feature shows a larger pull-off force at high cycling rates (LFHR). Based upon our SEM inspection, the contacts with LFLR features usually have plastically flattened surfaces. However, for the samples found with LFHR features, surface damage has always been observed. These observations suggest that the LFLR mode is associated with brittle separation, and the LFHR mode is associated with ductile separation.

For a brittle separation, the magnitude of the pull-off force depends on the bonding strength at the interfaces. The longer time in contact, the stronger bonding could develop, which in turn leads to a larger pull-off force to separate the contacts. Observed LFLR features could be due to the contact time and load effects on the interfacial bonding strength.

A simple calculation shows a possible surface film effect in the LFLR mode. Assuming elastic separation in LFLR mode, the pulloff force $F_{\text {pulloff }}$ can be estimated by using the JKR contact adhesion model:

$$
F_{\text {pulloff }}=1.5 \pi w R
$$

where $R$ is the radius of curvature of the contact bump, and $w$ is the work of the adhesion. In our contact test, we observed a plastic deformation region on top of the contact bump. Due to plastic deformation, the effective asperity radius of curvature $R_{\text {eff }}$ after elasto-plastic or fully plastic deformation is different than its initial value $R$. Correspondingly, the pull-off force can be expressed in term of $R_{\text {eff, }}$

$$
F_{\text {pulloff }}=1.5 \pi w R_{\text {eff }}
$$

Further assuming that all the plastic deformation occurs during the first cycle of loading, the unloading and the next cycle of loading are elastic. To estimate the effective radius of curvature after a single loading event, we use the Kogut-Etsion (KE) curvefit solutions from finite element analysis [6]. Based upon the KE model, the bump with original radius of curvature $R=15 \mu \mathrm{m}$, hardness $H=500 \mathrm{MPa}$, and loading force $200 \mu \mathrm{N}$, the effective radius of curvature $R_{\text {eff }}$ can be calculated as $45 \mu \mathrm{m}$. Using Equation (2), we calculated that the work of adhesion is $0.37 \mathrm{~J} / \mathrm{m}^{2}$ for $80 \mu \mathrm{N}$ pull-off force; the work of adhesion is $0.18 \mathrm{~J} / \mathrm{m}^{2}$ for $40 \mu \mathrm{N}$ pull-off force. These works of adhesion are much smaller than the work of adhesion between clean metal contacts $\left(1 \sim 3 \mathrm{~J} / \mathrm{m}^{2}\right)$. This calculation indicates that there are surface films between the gold contacts. These interposed films usually lower the surface energy and can keep the separation in the brittle mode.

The kinetics of forming or destroying the interposing surface film could contribute to the observed LFLR rate-dependent features. Since gold is a hydrophilic surface and the tests were all done in room air, such surface film effects could be due to the meniscus force effect.

For a meniscus effect, there is a time constant for forming meniscus bridges. The experiments by Szoszkiewicz [7] have shown that at $37 \%$ relative humidity, the mean meniscus nucleation time is around $0.7 \sim 4.2 \mathrm{~ms}$ at room temperature. For contact times less than the mean nucleation time, the number of menisci formed will be reduced. The longer the time in contact, the more menisci can be developed, hence the larger meniscus force. Our rate sweeping experiment shows that the pull-off force drops when the cycling rate is larger than $100 \mathrm{~Hz}$. This may indicate the equilibrium time for meniscus condensation is about $5 \mathrm{~ms}$, which is in the range of the meniscus nucleation time scales observed by Szoszkiewicz. Further increasing the cycling rate could cause some meniscus bridges to fail to nucleate.

Furthermore, we can verify that, in LFLR mode, the measured difference in pull-off force at two rates is of the same magnitude as the meniscus force effect. It was shown that the force difference 
between the high cycling rate and low cycling rate is in the range of $20 \sim 60 \mu N$. For a water thin film between a hemispherical bump and a flat surface, the meniscus force can be estimated by:

$$
F_{m}=4 \pi R \gamma_{l v} \cos \theta
$$

where $\gamma_{\mathrm{lv}}$ is surface tension of water film, equal to $72.7 \mathrm{dyn} / \mathrm{cm}$. Since there is an intrinsic layer of water thin film on a gold surface, the contact angle is chosen as zero. Using an effective radius of $45 \mu \mathrm{m}$, the meniscus force can be calculated as $40 \mu \mathrm{N}$, which is the force difference between $0.5 \mathrm{~Hz}$ and $300 \mathrm{~Hz}$. Both the transition time and the magnitude of the force suggest that the LFLR effect could be due to the meniscus force effect in brittle separation. The LFLR feature could be used to identify a brittle separation.

On the other hand, ductile separation is usually associated with bulk material stretching and nanostructure separation. Such nanostructure separation could activate viscous effects in the gold. Usually, for inelastic deformation in a metal, time-independent yield flow is the dominant deformation mechanism. The yield flow is activated by nucleation of dislocations, which defines the perfectly plastic behavior observed in macroscopic crystalline metal. However, when the structure scale reduces to the nanoscale, the dislocation sources are largely reduced. Under this situation, a time-dependent "creep” flow becomes dominant. In "creep” flow, diffusion is a dominant mass transport mechanism. The low barrier height for atom diffusion around the nanostructure allows atoms to move fast on the surface. Surface tension driven diffusion has been observed in the separation of $\mathrm{Au} / \mathrm{Au}$ [8] and $\mathrm{Pb} / \mathrm{Au}$ [9] nano junctions. Even though it is unrealistic to assume the nano junction is a liquid, it is believed that at the last stage of the separation, (when the neck is narrowing down to several nanometer), the junction behaves like a "liquid" [10].

Due to the "creep" flow in nanojunctions, we would expect a viscous effect in ductile separation. Such viscous effects could be the reason for observed LFHR features, which gives a larger pulloff force at a high cycling rate. In particular, we found that, by increasing the unloading rate, independent of the cycling rate, the pull-off force increased in the LFHR mode.

In ductile separation, the magnitude of the pull-off force is then related to the volume of the ductile area. The increase in the pull-off force in the LFHR mode may indicate an increase of the ductile area.

Two features in LFHR mode have been observed: (1) the force increase in LFHR mode is random. This could be due to a random distribution of the ductile area. (2) In the LFHR mode, a larger pull-off force is observed at large loading and a high unloading rate. This could be because larger loading can activate more ductile region and lead to larger viscous effects.

\section{CONCLUSION}

Two rate-dependent pull-off force features have been observed in gold micro contacts. One is a larger pull-off force at low rate (LFLR), and the other is larger pull-off force at high rate (LFHR). We found that the rate-dependent features could be related to two separation modes: brittle and ductile.

For LFLR features, the rate dependences may be due to surface effects during brittle separation. Longer contact times at lower rate can help to develop a larger surface force between contacts. Since all these tests were performed in room air with 30\% 40\% humidity, such surface effects could be due to menisci between contacts. We found that the measured force and rate features are consistent with the characteristics of meniscus condensation. SEM inspections show that the contact surfaces in LFLR usually are plastically flattened.
For LFHR features, the rate effects could be due to the viscous effects in ductile separation. During the ductile separation, there are nano tips drawn out of the surfaces. Surface tension driven diffusion in these nanostructures causes viscous effects during separation. The viscous effects can lead to a larger pull-off force at a higher unloading rate. We observed that the increase of pull-off force in the LFHR mode has considerable scatter. This could be due to the random nature of ductile separation. In general, once the ductile separation has been identified during the cycling test, the surface is found to be damaged. SEM inspection shows that the contact surfaces in LFHR mode are rough, with significant wear and sharp peaks characteristic of ductile separation.

The significance of this work is the identification of the ratedependence of the pull-off forces in metal contacts and its relation to the separation modes. By monitoring rate-dependent features during cycling tests, the separation mode can be identified in situ. This method can be a useful technique for metal contact microswitch reliability studies.

\section{REFERENCES}

[1] M. Rebeiz, J.B. Muldavin, "RF MEMS Switches and Switch Circuits”, IEEE Microwave Magazine, 12, 59 (2001).

[2] L. Chen, N.E. McGruer, and G.G. Adams, "AFM-Based Testing and Measurements of Contact and Stiction in a Micromechanical Switch” in Proc., ASME/STLE Inter. Joint. Tribol. Conf., Long Beach, California USA, TRIB 200464347 (2004).

[3] L. Chen, H. Lee, Z.J. Guo, N.E. McGruer, K.W. Gilbert, S.Mall, K.D. Leedy, and G.G.Adams, “ Contact Resistance Study of Noble Metals and Alloy Films Using a Scanning Probe Microscope Test Station”, Journal of Applied Physics, 102, 074910 (2007).

[4] N.E. McGruer., G.G. Adams, L. Chen, Z. Guo, and Y. Du, "Mechanical, Thermal, and Material Influence on OhmicContact-Type MEMS Switch Operation", in Proc. IEEE Microelectromech. Syst., Turkey, 1 (2006), pp. 230-233.

[5] Y. Du, L. Chen, N.E. McGruer, G.G. Adams, I. Etsion, “ A Finite Element Model of Loading and Unloading of an Asperity Contact with Adhesion and Plasticity”, Journal of Colloid and Interface Science, 312, 522 (2007).

[6] L. Kogut and I. Etsion, "Adhesion in Elastic-Plastic Spherical Microcontact”, Journal of Colloid and Interface Science, 261, 372 (2003).

[7] R. Szoszkiewicz and E. Riedo, "Nucleation Time of Nanoscale Water Bridges”, Physical Review Letter, 95, 135502 (2005).

[8] T. Kizuka, “ Atomic Process of Point Contact in Gold Studied by Time-Resolved High-Resolution Transmission Electron Microscopy”, Physical Review Letters, 81, 4448 (1998).

[9] L. Kuipers and J.W.M. Frenken, "Jump to Contact, Neck Formation, and Surface Melting in Scanning Tunneling Microscope”, Physics Review Letters, 70, 3907 (1993).

[10] N. Agraït, A.L. Yeyati, and J.M. van Ruitenbeek, “ Quantum Properties of Atomic-Sizes Conductors” Physics Reports, 377, 81 (2003). 